EPiC Series in Language and Linguistics
Volume 3, 2018, Pages 131-137
LSP in Multi-disciplinary contexts of
Teaching and Research. Papers from the
16th International AELFE Conference

\title{
Communication in virtual work: Towards a reliable framework for piloting training modules
}

\author{
Penny MacDonald, Carmen Pérez-Sabater, Begoña Montero-Fleta \\ Department of Applied Linguistics, Universitat Politècnica de València, Valencia (Spain)
}

\begin{abstract}
Improving the employability of graduates and professionals is the main aim of the European project CoMoViWo, a project which strives to address linguistic, virtual communication and intercultural challenges in global work contexts. To this end, the design and development of a series of intensive modules to improve communication in virtual and mobile contexts in the workplace is the objective of this three-year research and development project. To validate these modules, CoMoViWo has sought to establish a framework for the subsequent piloting of the materials. The present paper is delivered at the end of the third year of the project, and reports on the process of creating a questionnaire, a useful tool to pilot the communication learning modules. The rationale involved in the design and implementation of the questionnaire is discussed. The grid was designed following the recommendations proposed in several "Quality Guidelines" publications during recent years (see, for example, Lasnier et al., 2000). These criteria involve aspects such as transparency, reliability, integrity, practicality, attractiveness, flexibility, awareness and generativeness. The questionnaire was administered to the students to get feedback on the learning modules and their satisfaction with the modules. Although the students were not necessarily in a position to address the validity of the pedagogical aims of the input, their feedback on the structure, content, format and exercises/tasks, and their engagement with the platform and activities provided important data on their feasibility. The analysis of the results obtained indicate that the process of piloting the modules is an essential part of any materials elaboration project and vital for giving relevant feedback for the final stage of materials development. This confirms the validation of the grid devised and confirms the considerable relevance of the piloting process.
\end{abstract}

Keywords: Piloting, training modules; virtual communication; workplace language; intercultural communication.

\section{Introduction}

Communicating in virtual and multicultural environments is a skill increasingly demanded in current working environments. With this in mind, new projects such as CoMoViWo (Communication in Mobile and Virtual Work) are being developed. CoMoViWo is financed by the Education, Audiovisual and Culture Executive Agency (EACEA) of the European Union within the Erasmus+ Programme. The aim of the project was to develop a set of learning modules on the communication practices in digitally mediated professional environments to meet the demand of workplace communities. To this end, the project has addressed three key features of 
communication in the workplace: linguistic challenges, e.g., uses of a second language and lingua franca in a business setting; virtual communication challenges, e.g., how to be effective communicators both in face to face contexts and in virtual contexts; and intercultural challenges, e.g., how to accommodate different values and turn taking styles, and the interpretations of meaning that these might incur.

The learning materials created were planned and developed according to Quality Guidelines published during recent years (see, for example, Lasnier et al., 2000). These criteria involve aspects such as transparency, reliability, integrity, practicality, attractiveness, flexibility, awareness and generativeness. Key elements for assessing the quality of an online course, accessibility, clear organization of course content, clear expectations, and engaging communication, suggested by Richardson and Turner (2001) and Lee and Duncan-Howell (2007) are similar to the domains of our questionnaire.

A number of publications of the authors have described the different stages of the project completed so far (see, for example, Montero-Fleta et al., 2017). The final stage of the project, piloting, is the main focus of this article.

\section{Aims of the present research}

A final step of CoMoViWo was to ensure that the materials created met the objectives pursued, a necessary requirement to be carried out before editing the learning modules. The aims of this paper are twofold:

- Create a piloting grid to get feedback on the English and Spanish modules,

- Analyse the results obtained in the piloting carried out by the Universitat Politècnica de València (UPV), a partner of CoMoViWo.

\section{Research design}

To meet the aims above, research was devised in different phases: First phase: planning the piloting and assessment; Second phase: piloting the modules by students, partner HEIs and working life representatives.

\subsection{First phase: creating the grid}

This phase consisted in planning the piloting and the assessment of the final product by means of the creation of a grid that could provide accurate and reliable feedback on the adequacy of the learning modules. A survey tool was devised based on a careful review of the literature on best practices in online learning. Literature on the evaluation of online learning resources emphasises some key aspects that must be included in these assessment tools. Wright (2003) establishes a complete guide of criteria covering a wide range of aspects, from layout to course content. Wright's aspects include accessibility, organisation, the adequacy of the level of the language, the adequacy of the layout, of the content, and the correct establishment of goals and objectives, among many others. However, despite the great importance of these factors, Rothman et al.'s (2011) study on another similar long list of criteria concluded that the organisation and format of the content was a crucial aspect in order to attain positive evaluations of the online course.

In this case, some other characteristics of context-bound approaches are also being considered. Recommended by Hosie, Schibeci and Backhaus (2005) for resources in quality learning materials, these aspects include currency, if the age of the resources is adequate to the subject matter; richness, in other words, if they reflect a rich variety of perspectives; purposeful use of the 
media, which asks if media is suitable for the purpose intended; and finally inclusivity, that is, if materials reflect social, cultural, and gender inclusivity.

Consequently, these aspects had an important role in the grid developed by the UPV to assess the adequacy of the learning practices. Thus, it contained eight sections reflecting the aspects named above as traditionally related to the evaluation of online learning courses: structure, content, format, exercises, language level, video/audio, technical problems and observations. To guide the users in their answers, a total of eighteen questions on their satisfaction with the virtual training modules of CoMoViWo both in Spanish and English were included in the grid.

\subsection{Second phase: piloting}

The aim of the second phase was to make all the user groups test the feasibility of the material as well as to let them provide feedback on each unit in CoMoViWo.

Regarding the students' participation, thirty-seven students used the grid (see Table 1 in the Appendix) to assess all the material available on the virtual learning platform. The students involved in the piloting were undergraduate students, graduates, as well as international exchange students from different academic backgrounds such as language learning, management, engineering, communication or cultural studies depending on the partner's needs and expertise. Some of the students piloted both English and Spanish modules and others only English or Spanish. While studying the modules, they were expected to fill in the questions posed in the grid. The students were required to give their opinion on each unit, providing positive as well as negative comments on the content of each section included. For the sake of clarity, as student's feedback was to be used to modify the courseware as part of the project's quality initiatives, participants were told to state clearly which exercises they were referring to, especially when being critical or wanting to specify something in particular. Thus, the authors of the training modules were provided with direct feedback from the students on the material. The opinions were to be used to improve the quality of the exercises in each module.

To ensure higher quality involvement and to enhance motivation, their engagement in the assessment process accounted for two ECTS credits. An important concern was to ensure anonymity; thus, to maintain the piloters' privacy, and the confidentiality of their responses and information given, no users' identification was downloaded.

Finally, this second phase of the piloting process also required the involvement of professionals in the field, 20 professionals from different institutions.

\section{Research design}

\subsection{Structure}

There was a general consensus on the organization and coherent structure of the material presented, as shown in the following answers (the opinions are copied as in the originals):

- "Everything was coherent and user friendly". "The order in this unit is coherent."

However, some students complained about the length of the texts to be worked on. They considered that shorter texts would make the reading more user-friendly.

\subsection{Content}

Most participants agreed on the interest and relevance of the units, as well as the clarity of the presentations. They appreciated the focus of the material on learning for future work purposes. 
- "The topics are interesting and objectives are clearly explained. Explanations are clear."

But despite this agreement on the high quality of the content, there were some students that felt somehow lost in some units, found the material boring, or demanded more interactivity, as shown in these comments.

- "More intercultural communication in mobile context needed" "The content is relevant but sometimes a little bit boring."

\subsection{Format}

In general, users were satisfied with the format, as shown in this comment.

- "Everything looked good and well organized".

However, a more colourful display was frequently demanded to make the modules more attractive. Also, for many of the users, a certain amount of consistency and standardization was lacking in the materials at this stage of development.

- "Everything fine. Nervertheless I should recommand you to adopt EXACTLY the same presentation for all modules $(1,2,3)$."

It is important to highlight the contradictory views on the same material shown by some participants. Find below dissimilar opinions given by two different students on the same content:

- "Colours are used in a consistent way" and the opposite by another student: "colours are not used in a consistent way".

- "For me the format is very good", and a not so favourable opinion given by another student: "it is disorganized."

These contradictory points will be commented on below.

\subsection{Exercises/tasks}

In general, participants agreed on the variety of exercises and on the clarity of the instructions provided. They generally gave a positive feedback on the level of difficulty of the exercises.

- "Tasks were interesting and unconventional. They weren't too hard so I liked them."

However, some students, who seemed to be more proficient and did not need so much practice, complained about the repetition of some contents. Some other students thought the level of the exercises was low, whereas some thought it was too difficult. Some students missed more grammar, especially students showing a lower language level: "Grammar needs to be explained more." This did not surprise us, as although the modules were aimed at B1/B2 users, not all of them really had such an entry level. Users were expected to have an average command of grammar and vocabulary, (except for vocabulary directly connected to online communication) but this was not always the case.

\subsection{Video}

There was a general consensus on the video recordings as a graphic material to accompany and support the activities:

- "Video had appropriate level, the sound was clear." Technical problems

There were apparently several teething problems with the platform and access to the materials. Piloters were not able to access some links or they stated it took too long to download. 


\subsection{Observations}

Although specific questions had not been included to cover all the aspects proposed in Hosie, Schibeci and Backhaus (2005) (it was thought a grid that was too long would discourage students from giving detailed answers) some of the piloters did make reference to the question of whether the media is suitable for the purpose intended:

-Video's youtube is always in good quality and actual" "I liked very much the TED videos."

Another aspect focused on, if the 'age' of the resources is adequate to the subject matter:

- "Interesting and really current nowadays, talking about social networks and online communication, it is our way to communicate" "Topics were relevant and taking modern social media into account, plus being a guide to it."

And also if materials reflect social, cultural and gender inclusivity. In the feedback on the modules no negative or positive comments were made on the latter, although a gender-inclusive perspective was aimed at. As regards the other points, feedback included the following:

- "It was interesting to know better the different type of person from different type of culture."

- "Interesting topic about the different culture of different country in relation with politeness."

All in all, the students' piloting has ensured that the levels are adequate and that the exercises can be completed successfully. The topics selected are attractive, motivating and interesting to learners.

In general, standardization was one of the suggestions addressed in the feedback that needed improvement.

\subsection{Feedback from professionals}

Professionals in the field seemed to agree on some strategies that would improve the materials piloted: it was recommended that all input be removed that does not explicitly lead to a focus on intercultural communication in online and mobile contexts; language use should emerge explicitly from context as not all the activities in the materials appear to do that; recommendations were proposed on providing more grammar-driven materials and focusing on the levels, i.e. looking at what is required of a B1/B2 user and what they should be capable of doing. Finally, a clear distinction between self-study and tutor-led activities was demanded.

\section{Conclusions and further reflections}

The grid provided the appropriate framework to guide the students through specific questions on the materials and was also open to the piloters' own observations. The piloting offered the students some clearly rewarding experiences where they felt they had a chance to contribute to the final version of the modules with their own views on the material created.

The results of the piloting process, from both students and professionals, coincide with the outcomes of Rothman et al.'s (2011) study, which found that organisation and format are what concerns piloters most. The emphasis on standardization was also a recurrent aspect shown in the evaluations.

However, there were some contradictory opinions on the evaluation of the modules, possibly because learners bring different perspectives to online language learning (Yang \& Chen, 2009). These contradictory opinions also lead us to wonder whether the expertise of students should be considered at the same level. Some of the differences of opinion among the piloters may be due to the learners' (lack of) experience with online courses, and also, for example, regarding format 
and colour, their ideas are most likely to be influenced by their own particular taste and preferences.

In addition, with regard to students' reflections concerning the difficulty level of the exercises (some said they were easy, others, difficult), we stress the fact that the main aim of the modules, in both English and Spanish versions, is not to teach students the language competences already addressed in tens of standard language learning books aimed at helping students to pass from one level to the next. The CoMoViWo materials have been developed to provide training in online communication in the workplace, taking into account the globalised world we live in and the amount of virtual communication carried out between workers from very different cultural backgrounds. Nevertheless, with the aim of tackling learner variability and varying levels, more external links can be included in order to provide users with more detailed grammar information and practice. The researchers involved in the project seem to agree that students were not necessarily in a position to address the validity of the pedagogical aims of the input, while the feedback from professionals has been decisive for redesigning specific aspects of our modules. The results obtained certainly do merit our attention if we are to produce a set of materials that learners and teachers will want to use beyond the lifetime of the project.

\section{Appendix}

Table 1. CoMoViWo piloting feedback.

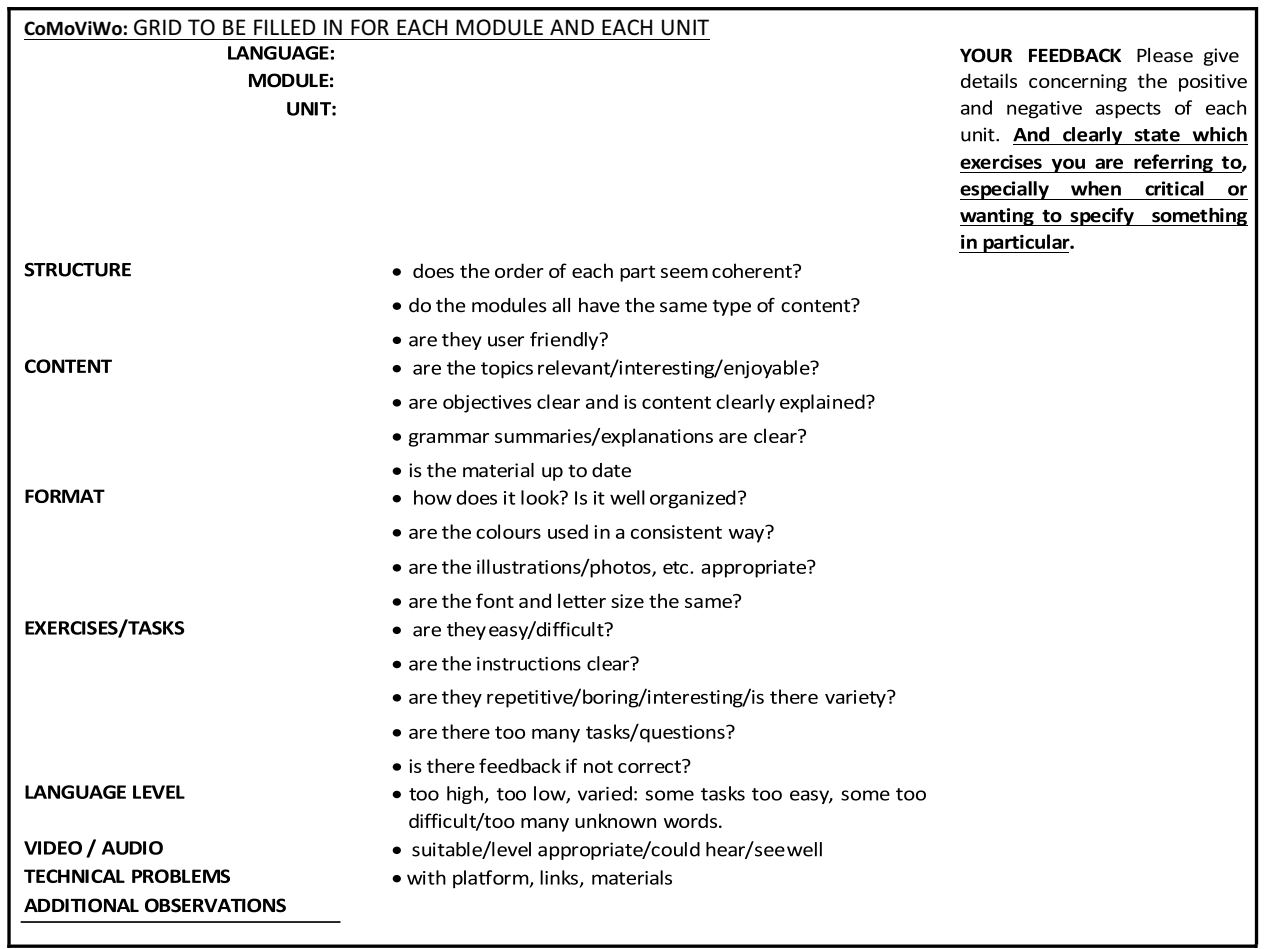

\section{References}

Cowling, J. D. (2007). Needs analysis: Planning a syllabus for a series of intensive workplace courses at a leading Japanese company. English for Specific Purposes, 26(4), 426-442. 
Hosie, P., Schibeci, R., \& Backhaus, A. (2005). A framework and checklists for evaluating online learning in higher education. Assessment \& Evaluation in Higher Education, 30(5), 539-553.

Lasnier, J. C., Morfeld, P., North, B., Serra Borneto, \& C. Spaeth, P. (2000). A quality guide for the evaluation and design of language learning and teaching programmes and materials. CD-ROM version.

Lee, K.T. \& Duncan-Howell, J. (2007). How do we know e-learning works? Or does it? E-Learning, 4(4), 482-496.

Pérez-Sabater, C., Montero-Fleta, B., Varhelahti Mervi, M., MacDonald, P., García-Carbonell, A., \& Conchado-Peiró, A. (2017). Hosting virtual group interactions: Language needs. ESP World, 52, 1-8.

Richardson, J. A., \& Turner, A. (2001). Collaborative learning in a virtual classroom: Lessons learned and a new set of tutor guidelines. In National Teaching and Learning Forum, 10(2), 7-9).

Rothman, T., Romeo, L., Brennan, M., \& Mitchell, D. (2011). Criteria for assessing student satisfaction with online courses. International Journal for e-Learning Security, 1(1-2), 27-32.

Wright, C. R. (2003). Criteria for evaluating the quality of online courses. Alberta Distance Education and Training Association. Available at elearning.typepad.com/thelearnedman/ID/evaluatingcourses.pdf

Yang, S. C., \& Chen, Y. J. (2007). Technology-enhanced language learning: A case study. Computers in Human Behavior, 23(1), 860-879. 